\title{
CONCEITOS DE CINÉTICA TUMORALAPLICADOS À QUIMIOTERAPIA PARA O CÂNCER DE MAMA
}

\author{
CONCEPTS OF TUMORAL KINETICS APPLIED TO CHEMOTHERAPY OF BREAST CANCER
}

Alexandre H. Macchetti ${ }^{1}$, Heitor R. C. Marana ${ }^{2}$, Míriam E. Cavallini ${ }^{3}$

\begin{abstract}
'Docente. Universidade Metodista de Piracicaba - UNIMEP. Pós-graduando. Departamento de Ginecologia e Obstetrícia. Faculdade de Medicina de Ribeirão Preto - USP. ${ }^{2}$ Docente. Faculdade de Medicina da Universidade de Ribeirão Preto -UNAERP. Médico Assistente. Departamento de Ginecologia e Obstetrícia. Hospital das Clínicas da Faculdade de Medicina de Ribeirão Preto - USP. ${ }^{3}$ Docente. Universidade Metodista de Piracicaba - UNIMEP.

Correspondência: Dr. Alexandre H. Macchetti - Rua D. Maria Jacinta, n 241 - sala 81. CEP: 13561-150. São Carlos - SP - Brasil. Tel/Fax: (16) 3376-3460 - E-mail: ahmacchetti@yahoo.com.br
\end{abstract}

Macchetti AH, Marana HRC, Cavallini ME. Conceitos de cinética tumoral aplicados à quimioterapia para 0 câncer de mama. Medicina (Ribeirão Preto) 2007; 40 (2): 213-22.

RESUMO: A maioria das pacientes com câncer de mama está sujeita à quimioterapia, e os comprovados benefícios da quimioterapia adjuvante na sobrevida global e livre de doença podem ser explicados pelos conceitos de eliminação celular. A aplicação dos conceitos de eliminação logarítmica de Skipper ao crescimento do câncer de mama humano, o qual parece seguir a cinética gompertziana, sugere que não apenas o uso de drogas sem resistência cruzada seja importante, mas a dose e a intensidade da quimioterapia administrada sejam variáveis clinicamente importantes que podem ser manipuladas para aumentar a sobrevida dos pacientes. Esta revisão da literatura apresenta alguns conceitos teóricos de cinética tumoral importantes para o entendimento de dados empíricos no campo da quimioterapia adjuvante do câncer de mama. A elaboração dos esquemas quimioterápicos é abordada com relação a dose e eficácia dos agentes componentes e quanto ao conceito de intensidade de dose da dose administrada, com aumento das doses e redução dos intervalos entre os ciclos objetivando a otimização, formulando esquemas de alta-dose e dose-denso. A hipótese de Norton-Simon, em que a terapia seqüencial poderia tornar o esquema de administração de agentes citotóxicos dose-densa e que estes esquemas teriam vantagens sobre os esquemas convencionais de administração de drogas também são discutidos.

Descritores: Neoplasias Mamárias. Quimioterapia. Cinética Celular. Cinética Tumoral. Modelo de Crescimento.

\section{1- INTRODUÇÃO}

Estimativas do Instituto Nacional do Câncer (INCa) mostram que em 2006 cerca de $48.930 \mathrm{mu}-$ lheres devem receber o diagnóstico de câncer de mama, sendo que aproximadamente $60 \%$ com estadio avançado $^{1,2}$. A implantação de programas de rastreamento para a detecção precoce do câncer de mama permite o aumento do número de cirurgias conser- vadoras, mas não impede que uma parcela considerável das mulheres ainda morra de câncer de mama recorrente. Este fato sugere a existência de micrometástases no momento do diagnóstico, para as quais a quimioterapia adjuvante é o único tratamento efetivo, seja prevenindo ou retardando a progressão da doença ${ }^{3}$.

A introdução de drogas com novos mecanismos de ação e em novas combinações busca a eli- 
minação destas micrometástases, pois o impacto da quimioterapia é de amplitude modesta no que diz respeito às recidivas, principalmente para os pacientes com o envolvimento metastático dos linfonodos axilares, ainda que este seja mínimo ${ }^{4}$.

O conhecimento dos conceitos teóricos de cinética tumoral é imprescindível a todos os profissionais e alunos que atuam na assistência ao paciente oncológico, pois permite o entendimento da história natural do câncer e do efeito administração das drogas citotóxicas sobre a proliferação das células neoplásicas, e por fim a compreensão dos dados dos recentes ensaios clínicos no campo da terapia adjuvante no câncer de mama que ratificam a importância dos modelos teóricos de crescimento tumoral.

Ensaios clínicos prospectivos de quimioterapia no câncer de mama e revisões acerca de conceitos teóricos de cinética tumoral foram selecionados por meio de pesquisa de artigos na língua inglesa nas bases de dados PubMed e MEDLINE com ênfase nos últimos 5 anos.

\section{2- MODELOS DE CRESCIMENTO E CON- CEITO DE ELIMINAÇÃO LOGARÍTMICA}

O entendimento do crescimento tumoral inicialmente esteve baseado no modelo de crescimento exponencial proposto por Skipper ${ }^{4}$. Os dados deste modelo advieram da observação de tumores experimentais, principalmente da leucemia murina L1210, que apresentavam um padrão de proliferação celular exponencial, ou seja, ocorria a duplicação celular contínua em uma unidade fixa de tempo. Este crescimento

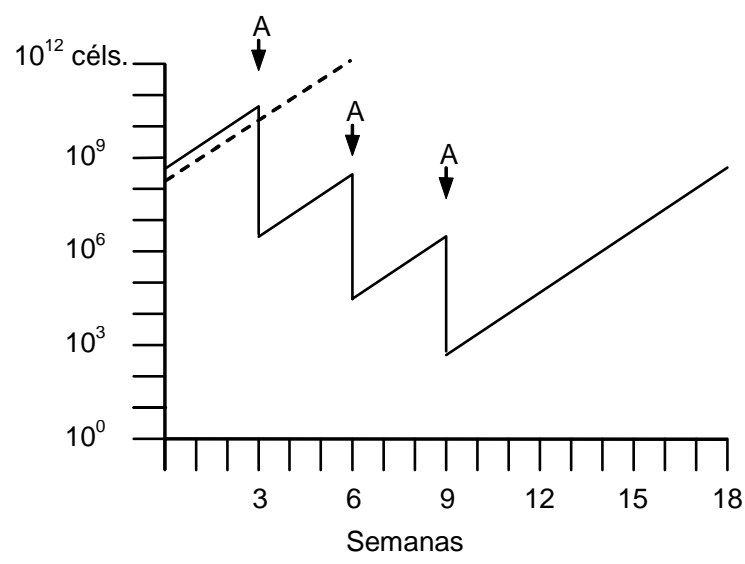

exponencial pode ser demonstrado graficamente por uma linha reta e crescente quando uma escala logarítmica representando o número de células tumorais é usada no eixo y, enquanto o tempo é representado em uma escala aritmética no eixo x (Figura 1). A extrapolação deste modelo de crescimento, típico das leucemias murinas, levou à estimativa de que $10^{12}$ células tumorais representariam o volume letal em um ser humano, e que o diagnóstico da recorrência da doença não seria realizado até que o valor atingisse algo entre $10^{10}$ e $10^{11}$ células tumorais.

Skipper também introduziu o conceito teórico de eliminação logarítmica ou log kill, no qual a administração de uma determinada dose de uma droga citotóxica eliminaria uma fração constante e não um número absoluto de células tumorais a cada ciclo ${ }^{4}$. Por este conceito uma população maior de células tumorais sofreria uma perda celular maior pela ação de uma determinada dose que uma população menor das mesmas células $5,6,7$.

Na Figura 1, assumindo-se $10^{11}$ células tumorais no momento da $1^{\mathrm{a}}$ administração da droga $\mathrm{A}$ e que esta gere a eliminação logarítmica de $4 \log$ a cada ciclo pode-se observar uma diminuição de $10^{11}$ para $10^{7}$ células, ou seja, uma diminuição de $9,99.10^{8}$ células. Assumindo-se que no intervalo entre as administrações das drogas ocorra a proliferação das células com acréscimo de $2 \log$, no momento da $2^{\mathrm{a}}$ administração da droga teríamos uma população de $10^{9}$ células que sob o efeito desta diminuiria para $10^{5}$ células, ou seja uma perda de $9,99.10^{7}$ células, menor comparando-se ao efeito da $1^{\mathrm{a}}$ administração à população inicial que era maior.

Figura 1: Ciclos sucessivos da droga $A$ interrompem o crescimento exponencial da subpopulação de células tumorais sensíveis à droga $\mathrm{A}$ conforme o conceito de eliminação logarítmica, e subpopulação de células tumorais resistentes à droga $A$ mantém 0 se crescimento exponencial. Não se conseguindo a eliminação completa das células tumorais após os ciclos, há o retorno da proliferação. 
Simulação matemática realizada por Day et al. estimou que em pacientes com câncer de mama com 10 ou mais linfonodos axilares acometidos por metástases a eliminação logarítmica variaria entre 6,5 e $9 \log$ a depender da intensidade de dose do ciclo administrado, um conceito a ser discutido adiante ${ }^{8}$. O efeito citotóxico da droga interrompe a proliferação exponencial das células a cada administração, mas a sua metabolização faz com que este efeito seja temporário, permitindo o recrudescimento da população, gerando a necessidade de sucessivas administrações para que ocorra a eliminação de toda a população (Figura 1) ${ }^{6 / 9}$.

A heterogeneidade celular é uma característica das neoplasias, sendo compostas por clones celulares com diferentes perfis de sensibilidade às drogas citotóxicas, o que possibilita a proliferação de um clone resistente à determinada droga $\mathrm{A}$, mantendo o seu crescimento exponencial (Figura 2). Na Figura 2 observa-se a simulação gráfica de resposta de um tumor composto por duas subpopulações com diferentes perfis de sensibilidade, e o efeito da associação de uma droga B para a qual teoricamente não haja resistência levando, em associação com a droga A, após sucessivas administrações, à eliminação logarítmica destes dois clones celulares, ratificando um dos princípios da combinação de quimioterápicos, que é a combinação de drogas com diferentes mecanismos de ação ${ }^{10,11,12}$.

Ensaios clínicos demonstraram que a administração de esquemas poliquimioterápicos é mais efetiva que a administração das drogas de forma isolada, principalmente quando elas são administradas na dose adequada, pois a combinação de dois ou mais agentes quimioterápicos pode freqüentemente, devido às suas toxicidades, levar à necessidade de redução da dose de cada um quando comparada às doses que seriam administradas isoladamente ${ }^{10,11,12}$.

Na composição de um esquema poliquimioterápico deve-se observar os perfis de toxicidade das drogas componentes, pois se eles forem diferentes podem conseguir a utilização destas em suas doses plenas $^{6,7}$. Compondo-se um esquema terapêutico no qual as doses de duas drogas, devido às suas toxicidades, necessitam ser reduzidas pela metade para que possam ser administradas combinadas, apenas poderá ser considerado mais eficiente se este obtiver uma eficácia maior do que $50 \%$ da eficácia das drogas administradas isoladas nas suas doses convencionais. Se isto não ocorrer, esta associação será menos eficaz, em termos de morte celular, do que $100 \%$ da droga $\mathrm{A}$ ou $100 \%$ da droga B, comprometendo a possibilidade de cura. Extrapolando-se este conceito, se duas drogas, A e B, podem ser administradas combinadas nas suas doses máximas isoladamente, então pode-se considerar esta combinação uma unidade aditiva $(\mathrm{A}+\mathrm{B})^{6,7}$.

Se duas combinações, $\mathrm{A}+\mathrm{B}$ e $\mathrm{C}+\mathrm{D}$, são consideradas duas unidades, para que se tente realizar a combinação de $\mathrm{A}+\mathrm{B}+\mathrm{C}+\mathrm{D}$ é necessária a redução da dose de $\mathrm{A}+\mathrm{B}$ em $50 \%$ e $\mathrm{C}+\mathrm{D}$ também em $50 \%$, os mesmos princípios de redução de eficácia explanados anteriormente devem ser aplicados 6,7 .

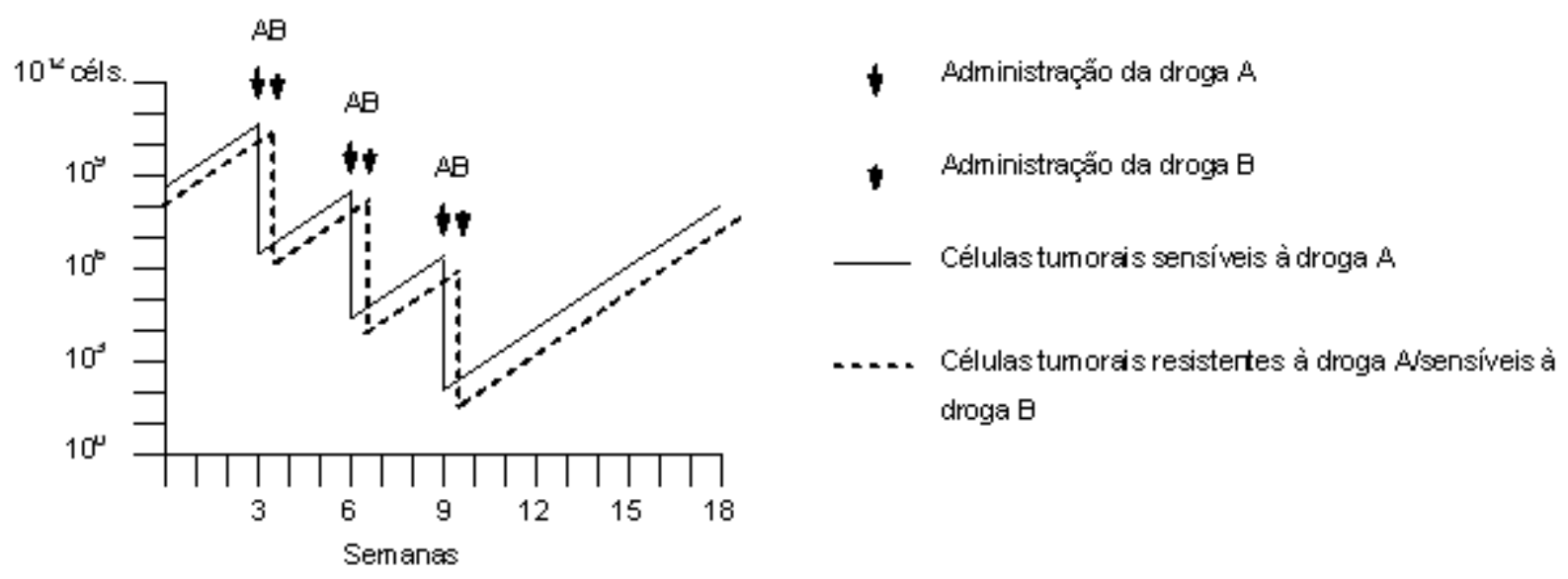

Figura 2: Administração de ciclos sucessivos da combinação $A+B$. Ocorre a interrupção do crescimento exponencial da subpopulação de células tumorais sensíveis à droga $A$, porém células resistentes à droga $A$, mas sensíveis à droga $B$ também têm a sua proliferação interrompida. Não se obtendo a eliminação completa das células tumorais após os ciclos, há o retorno da proliferação. 
A observação clínica, no entanto, mostra que a maioria dos tumores humanos, entre eles o câncer de mama, não segue o modelo de crescimento exponencial de Skipper, com tempo de duplicação fixo, mas um tempo de duplicação variável compreendido no modelo de crescimento de Gompertz, que graficamente descreve uma curva sigmóide ${ }^{13,14,15}$. Neste modelo de crescimento quanto menor o número de células tumorais menor o tempo de duplicação, ou seja, tumores menores têm maior velocidade de duplicação (Figura 3).

Na Figura 3 pretende-se representar a história natural de um câncer de mama, do surgimento da primeira célula até a detecção clínica do tumor com 1 $\mathrm{cm}^{3}$ e população estimada de $10^{9}$ células, com a proliferação celular seguindo o modelo de crescimento de Gompertz em um intervalo arbitrário de 72 meses.

Aplicando-se ao modelo de crescimento de Gompertz o conceito de log kill de Skipper observase que o impacto da terapia aplicada relaciona-se diretamente com a taxa de crescimento relativo: quanto menor o tumor maior a proporção de células eliminadas por uma determinada dose da droga A em relação à obtida em um tumor de maior tamanho ${ }^{13,14,15}$. A diminuição do número de células tumorais pelo esquema aplicado permite com que um tumor menor retorne ao crescimento entre os ciclos de quimioterapia com velocidade relativamente maior do que a observada previamente à terapia, observando-se um aumento da inclinação da curva de retomada de crescimento (Figura 3$)^{13,14,15}$.

A aplicação dos conceitos de cinética tumoral poderia explicar a diferença de eficácia entre dois esquemas de quimioterapia adjuvante agindo nas micrometástases ocultas gerando a diminuição do número de células neoplásicas. Na Figura 3 compara-se o efeito de 4 ciclos de 2 esquemas quimioterápicos, $\mathrm{A}$ e B, sendo que com o esquema B consegue-se obter uma maior redução no número de células tumorais a cada administração, ambos sem completa citorredução, mas que permitem explicar a diferença na sobrevida livre de doença.

A incorporação de drogas com mecanismos de ação cada vez mais eficientes e específicos na quimioterapia adjuvante permite que as micrometástases tenham, ao menos em teoria, seu número cada vez mais reduzido e levando a um maior intervalo tempo até a recorrência da doença. Conceito recente mostra que alterações na dose e nos intervalos entre as administrações das drogas já podem melhorar a eficácia. É o conceito de intensidade de dose, descrito a seguir.

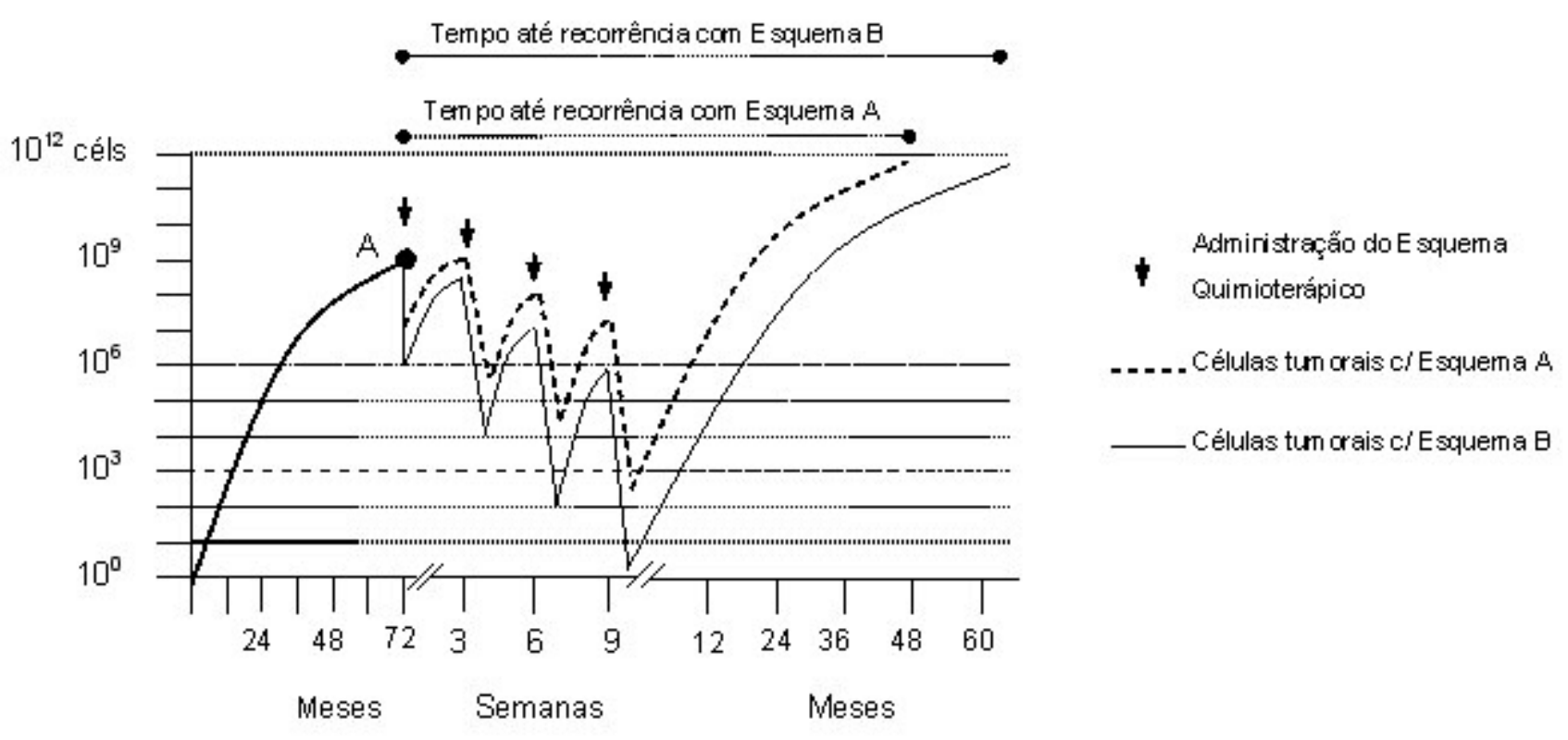

Figura 3: A proliferação de células tumorais conforme curva sigmoidal do modelo de crescimento de Gompertz é interrompida aos 72 meses quando com $10^{9}$ células o tumor clinicamente é detectado (ponto A). Simula-se a eficácia de dois esquemas quimioterápicos com base na diferença da eliminação logarítmica obtida por cada um. Após sucessivos ciclos destes dois esquemas quimioterápicos, o esquema $B$ consegue maior eliminação de micrometástases, mas ambos não conseguem a eliminação completa permitindo a proliferação destas gerando intervalos livres de doença diferentes. 


\section{3- CONCEITO DE INTENSIDADE DE DOSE}

O conceito de intensidade de dose foi introduzido por Hryniuk et al. (1998) e definido como a divisão da dose total da droga administrada por área de superfície corporal pela duração do tratamento, sendo expressa por $\mathrm{mg} / \mathrm{m}^{2}$ por semana, ou seja, uma função relativa da dose e freqüência da administração ${ }^{16,17}$.

Vários estudos mostram que a intensidade de dose está intimamente relacionada à eficácia do esquema quimioterápico, e o aumento da intensidade de dose pode ser obtido tanto pelo aumento absoluto da dose de uma droga ou de uma combinação preservando-se o intervalo de tempo entre as administrações (esquema high dose), quanto se mantendo as doses máximas permitidas devido à toxicidade com a diminuição dos intervalos entre as administrações (esquema dose dense $)^{18 / 24}$. A Figura 4 esquematiza estas combinações.

Inicialmente, a forma utilizada para o aumento da intensidade de dose foi o aumento progressivo na dose das drogas e verificando-se a eficácia dos esquemas (dose escalation), pois pelo conceito de eliminação logarítmica uma dose maior que a convencional geraria uma perda percentual ainda maior de células $^{18 / 24}$.

Estudos clínicos comprovam estes modelos, pois se verifica que há uma dose a partir da qual não há a melhora do tempo de sobrevida global ou livre de doença. No estudo HEPI 010, no qual se comparou, em pacientes com câncer de mama metastático, as respostas ao esquema composto por 5-Fluoracil, Epirrubicina e Ciclofosfamida com dose de Epirrubicina de $100 \mathrm{mg} / \mathrm{m}^{2}$ (FEC 100) versus esquema com dose de $50 \mathrm{mg} / \mathrm{m}^{2}$ (FEC 50), verificou-se um aumento de $16 \%$ na taxa de resposta a favor do esquema FEC100. No estudo FASG-05 estendendo-se este benefício à terapia adjuvante de pacientes com câncer inicial e axila comprometida, verificou-se que tanto a sobrevida global quanto à sobrevida livre de doença aumentam com a utilização do FEC 100, sem que se observe o acréscimo de cardiotoxicidade ${ }^{19,20}$.

No entanto, a partir de determinada dose, o aumento da concentração plasmática da droga não resulta em aumento da concentração no compartimento tumoral, observando-se que o aumento progressivo da dose passa a não estar necessariamente associado a uma maior eliminação de células tumorais e teoricamente a melhores resultados ${ }^{18,24}$.

Assumindo-se a ausência de interações com outras drogas ou alterações individuais do metabolis- mo do pacientes, a determinação da dose adequada pode ser ilustrada por meio do modelo $\mathrm{E}_{\max }$, utilizado freqüentemente para descrever o efeito farmacodinâmico das drogas, e expresso pela equação logística: $E=E_{\max } x C^{S} /\left(C^{S}+E C_{50} S\right)$, onde $E_{\max }$ é o efeito máximo, C é a concentração, $\mathrm{EC}_{50}$ é a concentração quando $50 \%$ do efeito máximo é conseguido, e 's' é o coeficiente de Hill que confere a forma sigmoidal à curva.

O gráfico que descreve a equação do modelo $\mathrm{E}_{\text {max }}$ apresenta uma escala de resposta e uma escala de concentração e descreve uma curva sigmoidal (Figura 4), o que pode ser traduzido como um benefício máximo com determinada dose e acima desta apenas aumento da toxicidade, sem qualquer aumento de eficácia ${ }^{19,24,25}$.

Bastholt et al. estudaram a correlação entre eficácia e toxicidade da monoterapia com epirrubicina administrada às pacientes com câncer de mama metastático com doses de 40,60, 90, ou $135 \mathrm{mg} / \mathrm{m}^{2}$, e verificaram que até $90 \mathrm{mg} / \mathrm{m}^{2} \mathrm{o}$ aumento progressivo da dose leva a melhores taxas de resposta e maiores períodos de controle da doença, enquanto doses maiores geraram apenas maior toxicidade ${ }^{21}$.

O estudo CALGB 8541 avaliou a eficácia de diferentes intensidades de dose do esquema adjuvante composto por Ciclofosfamida, Doxorrubicina e 5Fluoracil (CAF) na sobrevida global e livre de doença em pacientes com câncer de mama estadio $\mathrm{II}^{26}$. As pacientes foram randomizadas para receberem no dia um de cada ciclo de 28 dias, com a administração repetida de 5-Fluoracil no dia 8, o esquema CAF em altas doses (Ciclofosfamida $600 \mathrm{mg} / \mathrm{m}^{2}$, Doxorrubicina $60 \mathrm{mg} / \mathrm{m}^{2}$ e 5 -Fluoracil $600 \mathrm{mg} / \mathrm{m}^{2}$ ) por 4 meses, moderadas doses (Ciclofosfamida $400 \mathrm{mg} / \mathrm{m}^{2}$, Doxorrubicina $40 \mathrm{mg} / \mathrm{m}^{2}$ e 5 -Fluoracil $400 \mathrm{mg} / \mathrm{m}^{2}$ ) por 6 meses ou baixas doses (Ciclofosfamida $300 \mathrm{mg} / \mathrm{m}^{2}$, Doxorrubicina $30 \mathrm{mg} / \mathrm{m}^{2}$ e 5 -Fluoracil $300 \mathrm{mg} / \mathrm{m}^{2}$ ) por 4 meses. O esquema CAF com altas doses tinha o dobro da intensidade de dose e o dobro da quantidade das drogas do esquema com baixa dose, e o esquema CAF em moderadas doses tinha dois terços da intensidade de dose do grupo com altas doses, mas a mesma quantidade de droga.

Os grupos de pacientes submetidas ao esquema CAF com altas e moderadas doses apresentaram sobrevida global (SG) e sobrevida livre de doença (SLD) superiores às observadas no esquema com baixas doses (SG: $79 \% \pm 2 \%$ e $77 \% \pm 2 \%$ vs. $72 \% \pm 2 \%$; SLD: $66 \% \pm 2 \%$ e $61 \% \pm 2 \%$ vs. $56 \% \pm 2 \%$ ), mas nenhuma diferença estatística foi observada entre os esquemas com altas e moderadas doses ${ }^{26}$. 


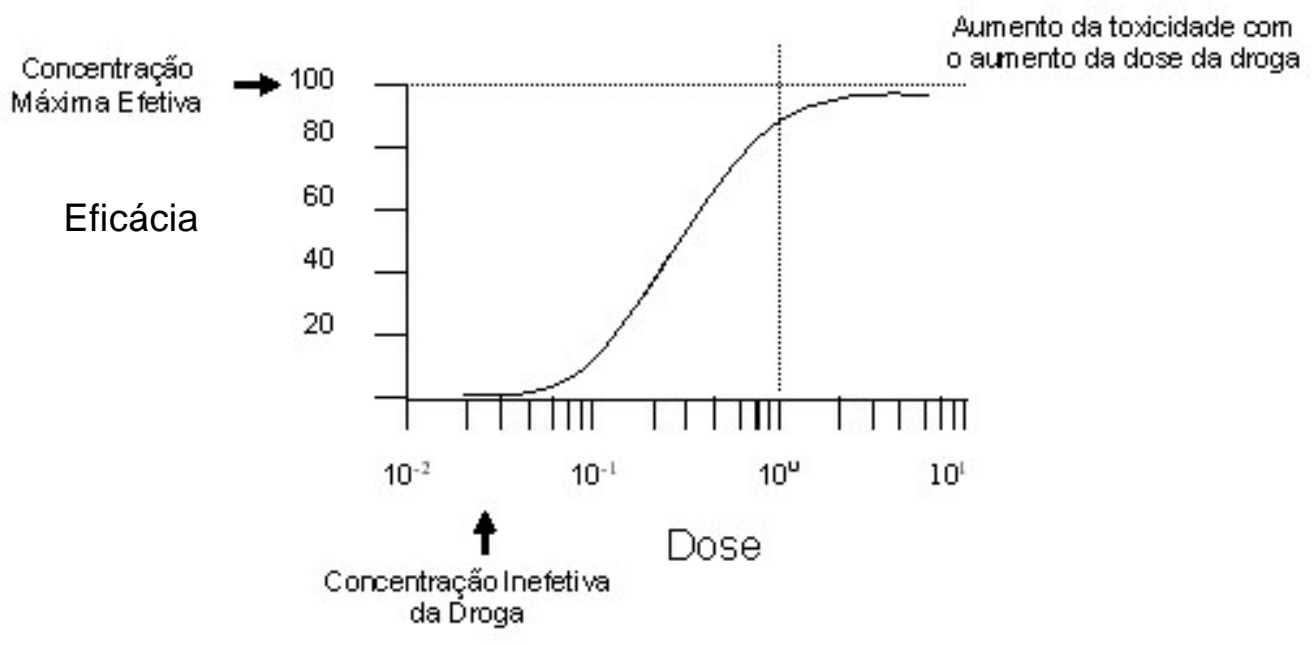

Figura 4: $\mathrm{O}$ modelo $\mathrm{E}_{\max }$ representa a concentração efetiva com relação à eficácia da droga citotóxica.

Ainda, se a partir de determinadas doses não se obtem melhora na eficácia, a utilização de esquemas com intensidade de dose inferior à convencional, seja por diminuição de dose ou por aumento do intervalo entre os ciclos devido à toxicidade, está fortemente associada à diminuição de sobrevida global e intervalo livre de doença. Os estudos de Bonadonna et al. mostraram que a utilização do esquema adjuvante com 12 ciclos da combinação de Ciclofosfamida, Metotrexate e Fluoracil (CMF) em doses menores que $85 \%$ da dose convencional associou-se a uma diminuição de $16 \%$ na sobrevida livre de doença e de $19 \%$ na sobrevida global após 30 anos de seguimento 27,28 .

Assim pode-se concluir que a dose e a intensidade de dose do esquema quimioterápico administrado são variáveis clinicamente importantes que podem ser manipuladas com o objetivo de melhorar a sobrevida global e intervalo livre de doença em pacientes com câncer de mama.

\section{4- HIPÓTESE DE NORTON-SIMON}

Os esquemas poliquimioterápicos podem ser classificados como alternantes ou seqüenciais, conforme a forma de administração $0^{6,7,29,30}$. Uma terapia composta por duas drogas ou combinações de drogas que possam ser consideradas uma unidade aditiva, $\mathrm{A}$ e $\mathrm{B}$ ou $\mathrm{A}$ e $\mathrm{C}+\mathrm{D}$, em suas doses plenas, pelo mesmo número de ciclos, e com intervalos fixos de administração podem ser administradas tanto de uma forma seqüencial, quanto de uma forma alternante, com di- ferentes impactos sobre a eficácia segundo a hipótese de Norton-Simon ${ }^{30 / 35}$. Nos esquemas alternantes a unidades componentes são administradas de forma alternada, ou seja, B após A e novamente A, ou A após $\mathrm{C}+\mathrm{D}$ e novamente $\mathrm{A}$. Nos esquemas seqüenciais cada unidade componente é administrada de forma sucessiva, ou seja, A após A por um determinado número de ciclos e depois B após B por um determinado número de ciclos $6,7,29,30$.

A administração dos quimioterápicos de forma seqüencial gera maior densidade de dose das drogas componentes em relação ao esquema alternante, pois se consegue administrar a dose total da droga A em um menor intervalo de tempo, ou seja, há densificação da dose da droga A e conseqüente aumento da intensidade de dose pelo menor intervalo de tempo ${ }^{6,7,29,30}$. $\mathrm{Na}$ terapia alternante com $\mathrm{A} \mathrm{e} \mathrm{B}$, os intervalos entre as administrações tanto de $\mathrm{A}$, quanto de $\mathrm{B}$, são maiores do que na terapia sequiencial, conferindo uma menor densidade para ambas as drogas 6,7,29,30.

A hipótese de Norton-Simon postula que os esquemas quimioterápicos dose-densos, com curtos intervalos entre os ciclos, resultariam em maior eficácia do tratamento, ou seja, incorpora aos conceitos existentes a possibilidade da forma de administração do esquema quimioterápico estar relacionada à eficácia do tratamento ${ }^{6,7,29,30}$. Segundo a hipótese de NortonSimon no esquema seqüencial, enquanto as células sensíveis à droga A são eliminadas depois de repetidos ciclos de terapia com a droga $\mathrm{A}$, as células sensíveis à droga $\mathrm{B}$ cresceriam sem impedimento até que 
o regime dose denso da droga $\mathrm{B}$ fosse introduzido ( $\mathrm{Fi}$ gura 5A). Na terapia alternante, onde o intervalo entre as administrações da droga A é maior, já que no intervalo a droga B é administrada, ocorrerá a proliferação das células sensíveis à droga $\mathrm{A}$, e no intervalo seguinte o mesmo ocorre com as células sensíveis apenas à droga $\mathrm{B}$, permitindo que ambas as populações escapem do potencial máximo de eliminação celular (Figura 5B).

O modelo de Norton-Simon prediz que a melhor forma de destruir um tumor composto por células heterogêneas é erradicar inicialmente as células numericamente dominantes, de crescimento mais rápido, seguido pela erradicação das células de crescimento mais lento, células resistentes, com uma segunda droga 6,7,29,30.

Estudo do Instituto Nacional do Câncer de Milão comparou a quimioterapia adjuvante com 4 ciclos
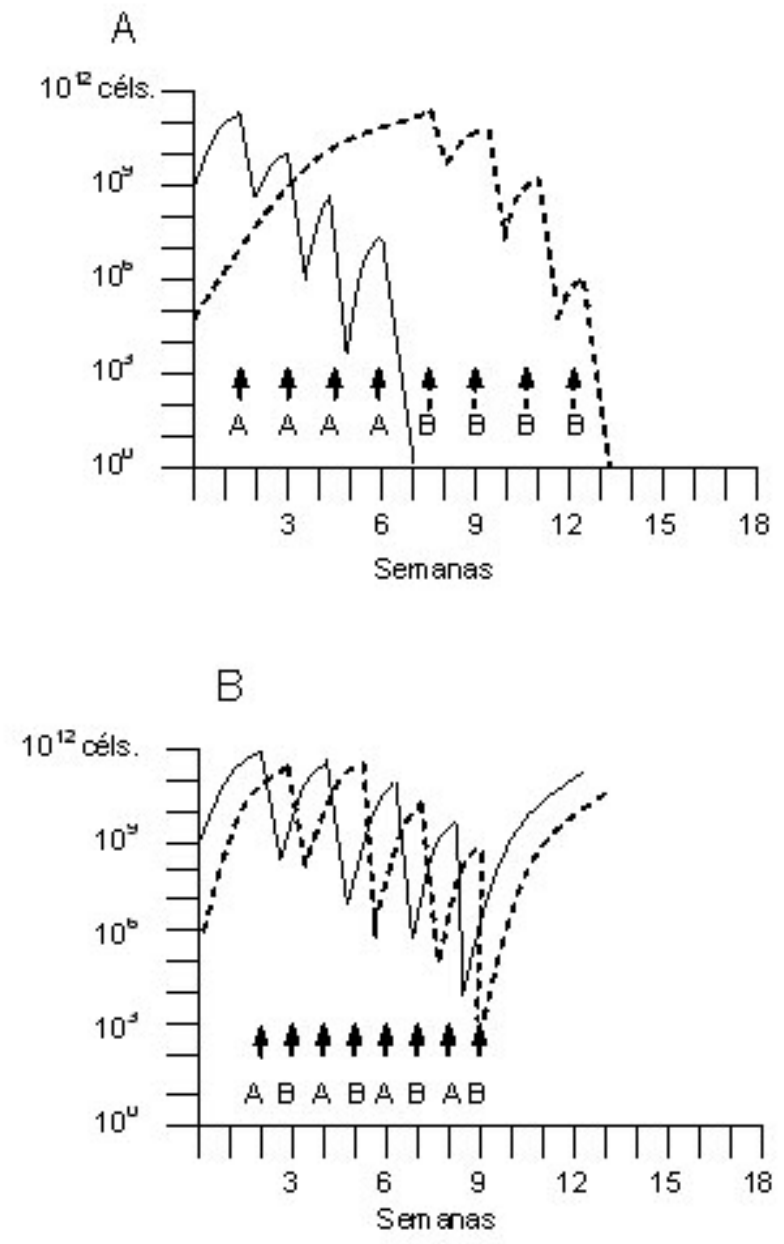

Figura 5. Hipótese de Norton-Simon aplicada sobre o conceito de eliminação logarítmica no crescimento gompertziano, assumindo a superioridade da terapia seqüencial dose-densa $(A)$ em relação à terapia alternante $(B)$ na citorredução de micrometástases. de doxorrubicina mais 8 ciclos da combinação Ciclofosfamida/Metotrexate/5-Fluoracil em pacientes com câncer de mama com 3 ou mais linfonodos axilares com metástases, administradas de forma seqüencial ou alternante. Ambos os esquemas tiveram o mesmo número de ciclos, intervalos de administração e as drogas as mesmas doses (Figura 6). O esquema seqüencial apresentou-se superior em termos de sobrevida global e livre de doença, concluindo que a administração da doxorrubicina em 9 semanas ao invés de 27 semanas (encurtamento do intervalo sem alteração da dose) gera o aumento da intensidade de dose de doxorrubicina e permite melhores resultados ${ }^{27,28}$.

No estudo CALGB 9741, pacientes com câncer de mama com linfonodos axilares sem metástases foram submetidas à quimioterapia adjuvante com doxorrubicina, ciclofosfamida e paclitaxel ${ }^{31,32,33}$. De forma randomizada formaram-se 4 grupos com diferentes formas de administração destas drogas: administração de doxorrubicina, paclitaxel e ciclofosfamida de forma seqüencial, a combinação doxorrubicina/ ciclofosfamida e paclitaxel de forma seqüencial, doxorrubicina, paclitaxel e ciclofosfamida de forma sequiencial com intervalos de 2 semanas entre os ciclos, e a combinação doxorrubicina/ciclofosfamida e paclitaxel de forma seqüencial e com intervalos de 2 semanas entre os ciclos (Figura 7). Objetivou-se avaliar a eficácia de esquemas com diferentes densidades de dose sobre a sobrevida global e livre de doença. Os resultados mostraram que houve diferença de sobrevida livre de doença e sobrevida global entre os esquemas seqüencial ou combinado, mas independente de outros fatores prognósticos, houve melhora em ambos os subgrupos com regimes mais densos $31,32,33$.

Quando a quimioterapia é administrada ela age não apenas nas células neoplásicas, que se dividem rapidamente, mas também nas células normais do corpo, particularmente nas células que tem alta taxa de proliferação como os folículos pilosos, as células da mucosa bucal e intestinal, e as células sangüíneas. As células-tronco da medula óssea, a partir das quais surgem as células sangüíneas circulantes, estão em constante maturação, e neste processo são mais sensíveis ao efeito do agente quimioterápico, ocorrendo um período de leucopenia, cujo ponto mais baixo é denominado nadir, e transitoriamente ocorre entre 7 e 14 dias após o último dia do ciclo. Um novo ciclo quimioterápico deve ser postergado até que a contagem absoluta de neutrófilos seja maior que $1500 / \mathrm{mm}^{3}$, pois o paciente está sujeito a mais infecções no período anterior a esta recuperação da medula óssea, o que ocorre em 
Doxormbicina $60 \mathrm{mgin}^{2}$

Seqüência

Seqüencial Dose Denso

Combinado

Combinado Dose Denso
P aditaxel $175 \mathrm{mg}^{2} \mathrm{~m}^{2}$

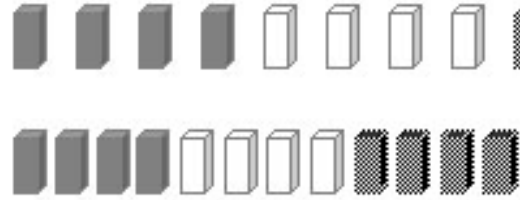

10

36 semanas

24 semanas

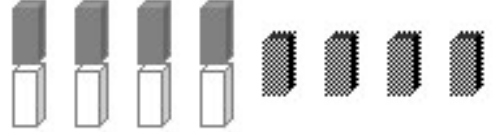

24 semanas

Figura 6: Grupos do estudo do Instituto Nacional do Tumor de Milão, nos quais se observa a intensidade de dose maior para a Adriamicina no esquema seqüencial.

Alternante
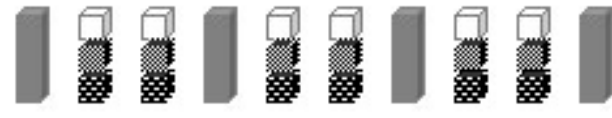

Seqüencial

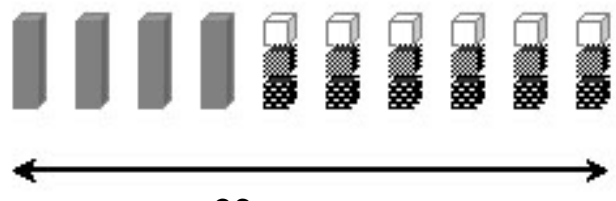

36 semanas

\section{Doxorrubicina $60 \mathrm{mg}^{2} \mathrm{~m}^{2} \in$ Cidofostanida $600 \mathrm{mg}^{2} \mathrm{~m}^{2}$ \\ Metotrexale $60 \mathrm{mgin}^{2}$ \\ 5-Fluoracil $600 \mathrm{mg} / \mathrm{m}^{2}$}

do fator de crescimento de colônias de granulócitos, mas estudo semelhante de Schwartz et al. utilizando esquemas convencionais e dose densos das combinações Adriamicina e Ciclofosfamida seguidos por Docetaxel ou Paclitaxel, ambos em sequiência, mostraram que embora as taxas de neutropenia possam ser diminuídas, a necessidade de transfusões sangüíneas e uso de eritropoietina aumenta, e a toxicidade cutânea grau 3 pelo esquema dose denso com uso de docetaxel limita a sua utilização na prática clínica, com aumento de $11 \%$ para $70 \%$ em relação ao esquema convencional ${ }^{36}$.

Assim, podemos concluir que embora as melhores taxas de resposta, au21 a 28 dias. Este efeito adverso da quimioterapia gera a necessidade do intervalo entre os ciclos de 21 a 28 dias. Quando se busca o aumento da intensidade da dose com a diminuição dos intervalos entre os ciclos para 14 dias, por exemplo, a recuperação fica comprometida gerando a necessidade de medicações de suporte como a eritropoetina e a filgrastima, também denominada fator de crescimento de colônias de granulócitos ${ }^{34,35,36}$.

A toxicidade hematológica dos esquemas mais densos pode ser minimizada com a administração mento de sobrevida global e livre de doença provenientes de ensaios clínicos com esquemas quimioterápicos de maior intensidade de dose ratifiquem os conceitos teóricos de cinética tumoral que os embasaram inicialmente, estes não diminuem a credibilidade de esquemas terapêuticos que utilizam doses convencionais, pois em muitos destes ensaios houve a necessidade de uso de fatores de crescimento, eritropoetina e antibióticos para os efeitos adversos junto ao aumento de internações, o que ainda limitam o uso destes esquemas à pesquisa clínica. 
Macchetti AH, Marana HRC, Cavallini ME. Concepts of tumoral kinetics applied to chemotherapy of breast cancer. Medicina (Ribeirão Preto) 2007;40(2): 213-22.

ABSTRACT: Most patients who have breast cancer are submitted to chemotherapy, and the proven benefits of adjuvant chemotherapy on disease-free and overall survival in breast cancer can be explained through concepts of cell kill. The application of log-kill concepts of Skipper to human breast cancer growth, which seems to follow Gompertzian kinetics, implies not only the use of non-cross-resistant drugs is important, but also the dose and the intensity of chemotherapy are clinically important variables which can be used in order to improve disease free survival and overall survival. This literature review presents certain important theoretical concepts of tumoral kinetics in breast cancer therapy. The chemotherapy is scheduled according to the dose and the compounded agent efficiency; and the dose-intensity concept with increasing of the doses and reduction of the breaks between the cycles aiming the optimization, formulating the high-dose and dose-dense schedules. Norton-Simon hypothesis, which supports sequential therapy might make the administration of cytotoxic agents into dose dense and these schedules would have an advantage on conventional schedules of drug administration which are also discussed.

Keywords: Breast Neoplasms. Chemotherapy. Cellular Kinetic. Tumoral Kinetic. Growth Model.

\section{REFERÊNCIAS}

1 - INCa. Instituto Nacional do Câncer. In: Estimativa 2006 - Incidência do Câncer no Brasil. <http://www.inca.gov.br/estimativa/2006/versãofinal.pdf>. Acesso em 16 de Setembro de 2006.

2 - Jones AL. Reduction in mortality from breast cancer. BMJ 2005;330(7485):205-6.

3 - Colleoni M, Rotmensz N, Peruzzotti G, Maisonneuve P, Mazzarol G, Pruneri G, Luini A, Intra M, Veronesi P, Galimberti V, Torrisi R, Cardillo A, Goldhirsch A, Viale G. Size of breast cancer metastases in axillary lymph nodes: clinical relevance of minimal lymph node involvement. J Clin Oncol 2005;23(7):137989.

4 - Skipper HE. Analysis of multiarmed trials in which animals bearing different burdens of L1210 leukemia cells were treated with two, three, and four drug combinations delivered in different ways with varying dose intensities of each drug and varying average dose intensities. Southern Research Institute Booklet 1986;420:87-92.

5 - Norton L. Kinetic concepts in the systemic drug therapy of breast cancer. Semin Oncol 1999;26(2):11-20.

6 - Norton L. Theoretical concepts and the emerging role of taxanes in adjuvant therapy. Oncologist 2001;6(3):30-5.

7 - Norton L. Conceptual and practical implications of breast tissue geometry: toward a more effective, less toxic therapy. Oncologist 2005;10(6):370-81.

8 - Day RS, Shackney SE, Peters WP. The analysis of relapsefree survival curves: implications for evaluating intensive systemic adjuvant treatment regimens for breast cancer. $\mathrm{Br} \mathrm{J}$ Cancer 2005;92(1):47-54.

9 - Takimoto $\mathrm{CH}$, Rowinski EK. Dose-Intense Paclitaxel: Dé jà Vu All Over Again? J Clin Oncol 2003;21(15):2810-4.
10 - Early Breast Cancer Trialists Collaborative Group (EBCTCG). Polychemotherapy for early breast cancer: an overview of the randomized trials. Lancet 1998;352:930-42.

11 - Carrick S, Parker S, Wilcken N, Ghersi D, Marzo M, Simes J. Single agent versus combination chemotherapy for metastatic breast cancer. Cochrane Database Syst Rev 2005; Artigo № CD003372.

12 - Jones D, Ghersi D, Wilcken N. Addition of drug/s to a chemotherapy regimen for metastatic breast cancer. Cochrane Database Syst Rev 2006; Artigo no:CD003368.

13 - Bajzer Z. Gompertzian growth as a self-similar and allometric process. Growth Dev Aging 1999;63(1-2):3-11.

14 - Dang C, Surbone A, Gilewski TA, Norton L. Cytokinetics. In: Kufe WD, Pollock RE, Weichselbaum RR, Bast RC, Gansler TS Holland JF, Frei E III, editors. Cancer Medicine. 6th. Hamilton: BC Decker Inc.;2003. p.645-68.

15 - Norton L. A Gompertzian model of human breast cancer growth. Cancer Res 1988;48(24):7067-71.

16 - Hryniuk W, Frei E III, Wright FA. A single scale for comparing dose-intensity of all chemotherapy regimens in breast cancer: summation dose-intensity. J Clin Oncol 1998;16(9): 313747.

17 - Skipper HE. Kinetics of mammary tumor cell growth and implications for therapy. Cancer 1971;28(6):1479-99.

18 - Budman DR. Dose and schedule as determinants of outcomes in chemotherapy for breast cancer. Semin Oncol 2004;31(15):3-9.

19 - Brufman G, Colajori E, Ghilezan N, Lassus M, Martoni A, Perevodchikova N, Tosello C, Viaro D, Zielinski C. Doubling epirubicin dose intensity $(100 \mathrm{mg} / \mathrm{m} 2$ versus $50 \mathrm{mg} / \mathrm{m} 2)$ in the FEC regimen significantly increases response rates. An international randomised phase III study in metastatic breast cancer. The Epirubicin High Dose (HEPI 010) Study Group. Ann Oncol 1997;8(2):155-62. 
20 - Bonneterre J, Roche H, Kerbrat P, Bremond A, Fumoleau P, Namer M, MJ, Schraub S, Fargeot P, Chapelle-Marcillac I. Epirubicin increases long-term survival in adjuvant chemotherapy of patients with poor-prognosis, node-positive, early breast cancer: 10-year follow-up results of the French adjuvant study group 05 randomized trial. J Clin Oncol $2005 ; 23(12): 2686-93$

21 - Bastholt L, Dalmark M, Gjedde SB, Pfeiffer P, Pedersen D, Sandberg E, M, Mouridsen HT, Rose C, Nielsen OS, Jakobsen $\mathrm{P}$, Bentzen SM. Dose-response relationship of epirubicin in the treatment of postmenopausal patients with metastatic breast cancer: a randomized study of epirubicin at four different dose levels performed by the Danish Breast Cancer Cooperative Group. J Clin Oncol 1996;14 (4):1146-55

22 - Orzano JA, Swain SM. Concepts and clinical trials of dosedense chemotherapy for breast cancer. Clin Breast Cancer 2005;6 (5):402-11.

23 - Seidman AD. Current status of dose-dense chemotherapy for breast cancer. Cancer Chemother Pharmacol 2005;56(1):78-83

24 - Gurney H. How to calculate the dose of chemotherapy. $\mathrm{Br} \mathrm{J}$ Cancer 2002;86(8):1297-1302.

25 - Mouton JW, Dudley MN, Cars O, Derendorf H, Drusano GL. Standardization of pharmacokinetic/pharmacodynamic (PK) PD) terminology for anti-infective drugs: an update. J Antimicrob Chemother 2005;55(5):601-7.

26 - Budman DR, Berry DA, Cirrincione CT, Henderson IC, Wood WC, Weiss RB, Ferree CR, Muss HB, Green MR, Norton L, Frei $E$ 3rd. Dose and dose intensity as determinants of outcome in the adjuvant treatment of breast cancer. The Cancer and Leukemia Group B. J Natl Cancer Inst 1998;90(16):1205-11.

27 - Bonadonna G, Moliterni A, Zambetti M, Daidone MG, Pilotti S, Gianni L, Valagussa P. 30 years' follow up of randomised studies of adjuvant CMF in operable breast cancer: cohort study. BMJ 2005;330(7485):217-20.

28 - Bonadonna G, Zambette M, Valagusa P. Sequential or alternating doxorubicin and CMF regimens in breast cancer with more than three positive nodes. JAMA 1995;273 (7): 542-7.
29 - Simon R, Norton L. The Norton-Simon hypothesis: designing more effective and less toxic chemotherapeutic regimens. Nat Clin Pract Oncol 2006;3(8):406-7.

30 - Fornier M, Norton L. Dose-dense adjuvant chemotherapy for primary breast cancer. Breast Cancer Res 2005;7(2):64-9.

31 - Citron ML. Dose density in adjuvant chemotherapy for breast cancer. Cancer Invest 2004;22(4):555-68.

32 - Piccart-Gebhart MJ. Mathematics and oncology: a match for life? J Clin Oncol 2003;21(8):1425-8.

33 - Citron ML, Berry DA, Cirrincione C, Hudis C, Winer EP, Gradishar WJ, Davidson NE, Martino S, Livingston R, Ingle JN, Perez EA, Carpenter J, Hurd D, Holland JF, Smith BL, Sartor $\mathrm{Cl}$, Leung EH, Abrams J, Schilsky RL, Muss HB, Norton $\mathrm{L}$. Randomized trial of dose-dense versus conventionally scheduled and sequential versus concurrent combination chemotherapy as postoperative adjuvant treatment of nodepositive primary breast cancer: first report of Intergroup Trial C9741/Cancer and Leukemia Group B Trial 9741. J Clin Oncol 2003;21(8):1431-9.

34 - Moore K, Crom D. Hematopoietic support with moderately myelosuppressive chemotherapy regimens: a nursing perspective. Clin J Oncol Nurs 2006;10(3):383-8.

35 - Rader M. Granulocyte colony-stimulating factor use in patients with chemotherapy-induced neutropenia: clinical and economic benefits. Oncology (Williston Park) 2006;20 (5 Suppl. 4):16-21.

36 - Schwartz J, Domchek SM, Hwang WT, Fox K. Evaluation of anemia, neutropenia and skin toxicities in standard or dosedense doxorubicin/cyclophosphamide (AC)-paclitaxel or docetaxel adjuvant chemotherapy in breast cancer. Ann Oncol 2005;16(2):247-52.

Recebido em 24/07/2006

Aprovado em 23/02/2007 